\title{
Putting the brakes on mammary tumorigenesis: Loss of STAT1 predisposes to intraepithelial neoplasias
}

\author{
Christine Schneckenleithner ${ }^{1}$, Zsuzsanna Bago-Horvath ${ }^{2}$, Helmut Dolznig ${ }^{3}$, Nina \\ Neugebauer ${ }^{1}$, Karoline Kollmann ${ }^{1}$, Thomas Kolbe ${ }^{4}$, Thomas Decker ${ }^{5}$, Dontscho \\ Kerjaschki², Kay-Uwe Wagner ${ }^{6}$, Mathias Müller, Dagmar Stoiber ${ }^{8,9}$ and Veronika \\ SexI $^{1}$ \\ ${ }^{1}$ Institute of Pharmacology and Toxicology, University of Veterinary Medicine, Vienna, Austria \\ ${ }^{2}$ Clinical Institute of Pathology, Medical University of Vienna, Austria \\ ${ }^{3}$ Institute of Medical Genetics, Medical University of Vienna, Austria \\ ${ }^{4}$ Biomodels Austria, University of Veterinary Medicine, Vienna and Biotechnology in Animal Production, IFA-Tulln, University \\ of Natural Resources and Applied Life Sciences, Vienna \\ ${ }^{5}$ Max F. Perutz Laboratories, Department of Genetics, Microbiology and Immunobiology, University of Vienna, Austria \\ ${ }^{6}$ Eppley Institute for Research in Cancer and Allied Diseases, University of Nebraska Medical Center, Omaha, Nebraska \\ ${ }^{7}$ Institute of Animal Breeding and Genetics and Biomodels Austria, University of Veterinary Medicine, Vienna, Austria \\ ${ }^{8}$ Ludwig Boltzmann Institute for Cancer Research, Vienna, Austria \\ ${ }^{9}$ Institute of Pharmacology, Medical University of Vienna, Austria \\ Correspondence to: Veronika Sexl, email: veronika.sexl@vetmeduni.ac.at
}

Keywords: Stat1, MIN, IRF 1, mammary cancer, tumorsurveillance

Received: December 1, 2011, Accepted: December 2, 2011, Published: December 19, 2011

Copyright: () Schneckenleithner et al. This is an open-access article distributed under the terms of the Creative Commons Attribution License, which permits unrestricted use, distribution, and reproduction in any medium, provided the original author and source are credited.

\section{ABSTRACT:}

Multiparous Stat1 $/-$ mice spontaneously develop mammary tumors with increased incidence: at an average age of 12 months, $55 \%$ of the animals suffer from mammary cancer, although the histopathology is heterogeneous. We consistently observed mosaic expression or down-regulation of STAT1 protein in wild-type mammary cancer evolving in the control group. Transplantation experiments show that tumorigenesis in Stat 1 $/-$ mice is partially influenced by impaired CTL mediated tumor surveillance. Additionally, STAT1 exerts an intrinsic tumor suppressing role by controlling and blocking proliferation of the mammary epithelium. Loss of STAT1 in epithelial cells enhances cell growth in both transformed and primary cells. The increased proliferative capacity leads to the loss of structured acini formation in 3D-cultures. Analogous effects were observed when Irf1 ${ }^{-/}$epithelial cells were used. Accordingly, the rate of mammary intraepithelial neoplasias (MINs) is increased in Stat1 $/-$ animals: MINs represent the first step towards mammary tumors. The experiments characterize STAT1/IRF1 as a key growth inhibitory and tumor suppressive signaling pathway that prevents mammary cancer formation by maintaining growth control. Furthermore, they define the loss of STAT1 as a predisposing event via enhanced MIN formation.

\section{INTRODUCTION}

The signal transducers and activators of transcription (STATs) are an intensely studied family of transcription factors that have been recognized as critical mediators of cytokine and growth factor receptor signaling, required for cell proliferation, survival and differentiation $[1,2]$.
Activation of STATs is frequently observed in different cancer entities and it has been postulated that deregulation of these factors may be involved in tumorigenesis. STAT1 is constitutively expressed throughout the entire development of the mammary gland. Its phosphorylation pattern - elevated in virgin glands, low throughout gestation and lactation, rising again at late involution - 
is unique, although the exact function of phosphorylation is unclear [3]. STAT1 is expressed in the epithelial compartment of the mammary gland, suggesting it has an active role in epithelial cells [4]. STAT1 is of particular interest in mammary cancer as it is known to possess an independent prognostic significance in human breast cancer: high activation of STAT1 has been reported to correlate with an overall longer and relapse-free survival $[5,6]$. Furthermore, treatment of human mammary tumor cells with cytostatic drugs has been shown to induce STAT1 activation, resulting in enhanced apoptosis [7, 8]. Recent work in mouse models of neu/ERBB2-induced breast cancer has underlined STAT1's tumor suppressive role [9, 10]. By crossing Stat ${ }^{\text {fl/l }}$ mice with MMTV-neuIRES-cre mice, Klover et. al. showed that tumor onset is significantly accelerated in Stat ${ }^{\text {flff }} x M M T V$-neu-IRES-cre mice compared to STAT1-expressing wild-type controls. This conclusion suggests that STAT1 has an autonomous role in neu/ERBB2-induced mammary tumor formation. The second report did not discriminate between the intrinsic and the immunological contribution of STAT1deficiency but came nevertheless to the conclusion that Stat $^{-/} x$ ERBB2/neu mice develop mammary tumors significantly faster than control mice. Together, the studies unequivocally defined STAT1 as a tumor suppressor in mammary cancer.

STAT1's tumor suppressing properties may be related to cell-intrinsic effects as STAT1 has been shown to block proliferation and to be involved in the induction of apoptosis [11-15]. Furthermore, Stat ${ }^{-/}$mice have a severely compromised immune system due to their lack of IFN-signaling $[16,17]$ as well as to an impaired cytotoxic NK-cell and CTL capacity [18, 19]. The contributions of these different components to mammary tumor surveillance are to date poorly understood. Moreover, all previous studies have been based on oncogene-driven mammary tumor formation in the absence of STAT1.

We now report for the first time that loss of STAT1 alone is sufficient to cause pregnancy-associated mammary cancer in BALB/c mice, independent of any other transgenic oncogene. By transplanting Stat ${ }^{-/}$ mammary glands into wild type recipient mice and vice versa we reveal that STAT1 contributes to the formation of mammary tumors through cell-intrinsic as well as immunological activities. Stat $1^{-/}$mammary epithelial cells exhibit enhanced proliferation, which might facilitate the development of mammary intraepithelial neoplasias (MINs) and subsequently also invasive mammary tumors. We suggest that STAT1/IRF1 acts in a linear axis to block growth. It is known that cytotoxic T-cells are impaired in Stat $^{-1-}$ mice and we also characterize cytotoxic T-cells as major mediators of mammary tumor surveillance.

\section{RESULTS}

\section{STAT1 deficiency is sufficient to cause mammary cancer}

$\mathrm{BALB} / \mathrm{c}$ mice are predisposed to develop mammary tumors and are therefore suitable to study spontaneous mammary tumorigenesis [11]. To evaluate the role of STAT1 in the spontaneous development of mammary tumors we crossed Stat ${ }^{-/-}$mice into the BALB/c genetic background. Groups of wild-type BALB/c and Stat $^{-/} / \mathrm{c}$ female mice were kept under breeding conditions and regularly controlled by palpation for mammary tumor formation. Within an average of one year, 55\% of the multiparous Stat $^{-/}$mice had developed mammary tumors. In the control group, only $10 \%$ had mammary tumors and disease onset was significantly later (Stat $1^{-/}$: 394.5 days \pm 13.52 and Stat $^{+/+}: 479.3$ days $\pm 11.46 ;{ }^{* *} P$ $=0.0089$; values represent mean $\pm \mathrm{SEM}$ ) (Fig. $1 \mathrm{~A}$ and 1B). No mammary tumors were detected in wild-type or Stat $^{-/}$nulliparous animals over a period of 20 months. Interestingly, all tumors that evolved in wild-type mice showed a mosaic expression and partial down-regulation of STAT1 protein (Fig. 1C). Loss or down-regulation of STAT1 was restricted to tumor cells and was not observed in the normal mammary tissue surrounding the cancer.

\section{Mammary epithelial transplantation uncovers a mammary gland cell-intrinsic and an immunological contribution of STAT1 to the development of mammary tumors}

To test whether these results stemmed from an intrinsic action of STAT1 within the mammary epithelium rather than simply being related to tumor-promoting changes triggered by the impaired immune system of the Stat $1^{-/}$animals, we transplanted Stat $1^{-/}$mammary epithelial tissue into the cleared fat pad of the $4^{\text {th }}$ mammary gland of Stat $^{+/+}$mice, and vice versa (see scheme in Fig. 1D). To control for tumor onset provoked by the transplantation procedure itself, we additionally transplanted Stat $1^{+/+}$ mammary tissue into Stat $^{+/+}$mice. Moreover, a group of Stat $^{-/}$mice was maintained under identical conditions in a non-transplanted setting. All animals were bred constantly to accelerate mammary tumor development. Animals were sacrificed when any evolving mammary tumor reached a diameter of one centimeter or earlier if there were signs of disease such as weight loss, scrubby fur or reduced mobility. All transplanted mammary glands - irrespective of whether a visible tumor had evolved - were subjected to histological analysis to assess the 
A

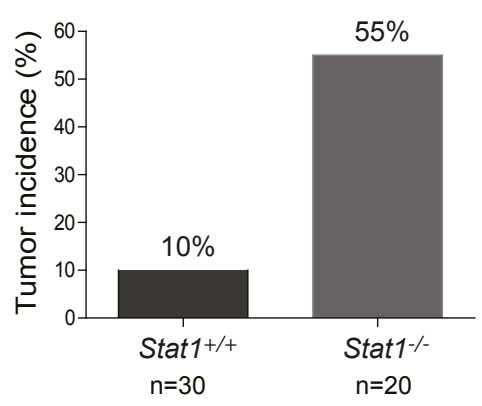

B

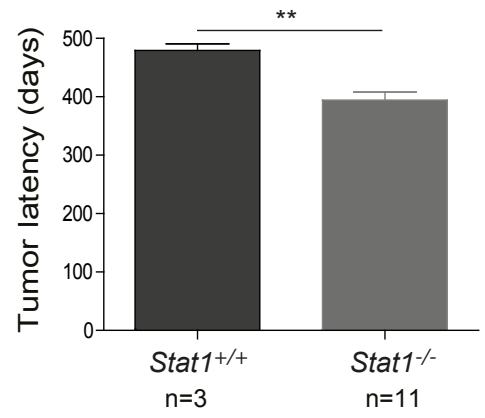

C

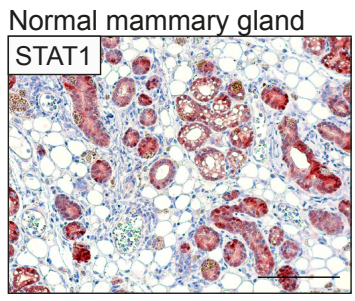

Stat $1^{+/+}$tumors

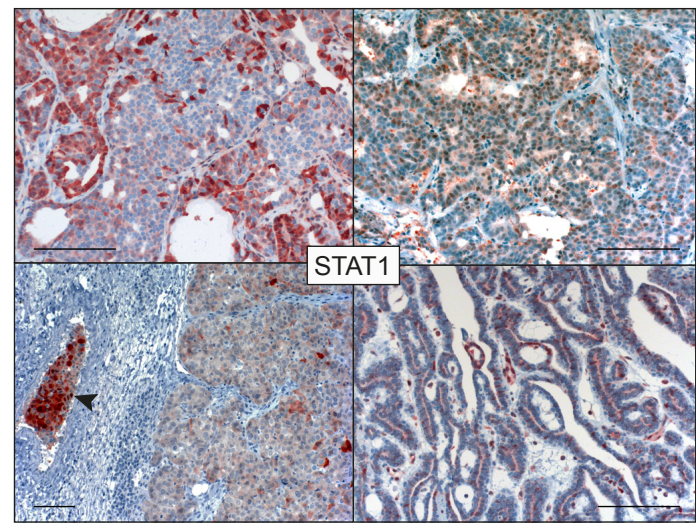

E

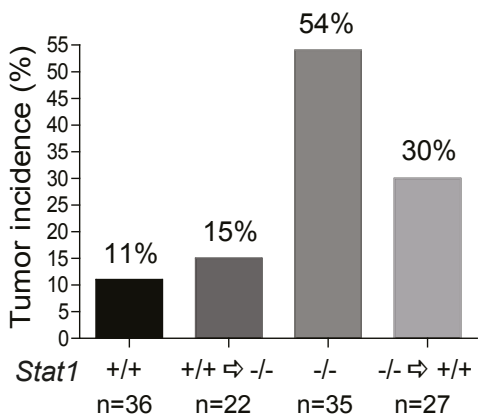

$\mathbf{F}$

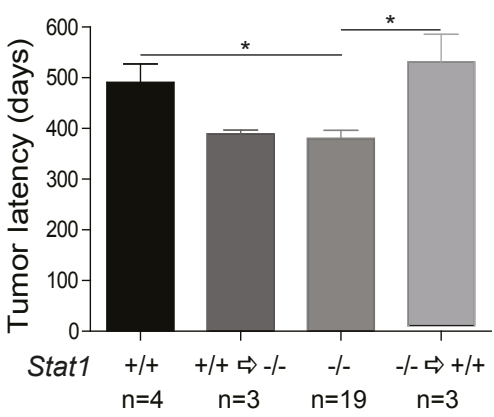

Figure 1: (A-C) Loss of STAT1 increases incidence and decreases latency of mammary tumors. (A) Incidence and (B) latency of spontaneously occurring, parity-induced mammary tumors in $\operatorname{Stat~}^{+++}(\mathrm{n}=30)$ and $\operatorname{Stat~}^{-/ \mathrm{H}}(\mathrm{n}=20)$ mice. Tumor development was monitored for up to 20 months. (C) STAT1-stained histological sections of a normal mammary gland from a multiparous wild type mouse after involution (upper panel) and of Stat ${ }^{+/+}$mammary tumors (bottom panel). Stat $1^{+/+}$tumors display mosaic expression and partial downregulation of STAT1 protein. The arrow indicates a vascular invasion. Scale bars: $100 \mu \mathrm{m}$. (D-F) Absence of STAT1 in the immune system and also in the mammary gland tissue influences mammary tumor formation. (D) Experimental setup of mammary gland transplantation experiment. Mammary gland tissue of Stat ${ }^{-1}$ mice was transplanted into the cleared fat pad of the $4^{\text {th }}$ mammary gland of Stat ${ }^{+/+}$mice $(\mathrm{n}=27)$, or Stat $^{+/+}$mammary glands were transplanted into Stat ${ }^{-/}$animals $(\mathrm{n}=22)$. Control groups: Stat $1^{+/+}$tissue transplanted into Stat $^{+++}$animals $(\mathrm{n}=9)$, non-transplanted Stat $^{-{ }^{-}}$animals $(\mathrm{n}=13)$. Mice were bred constantly and checked for mammary tumor growth for up to 20 months. On average, each animal had 7 litters. (E) Incidence and (F) latency of spontaneously occurring, parity-induced mammary tumors in all mice in the transplantation experiment. Tumors were classified into four groups: Stat $1^{++\mid}$tumors in Stat ${ }^{++\mid}$mice, Stat ${ }^{+/+}$tumors in Stat ${ }^{-/}$mice, Stat $1^{{ }^{-/}}$tumors in Stat1 ${ }^{-/}$mice and Stat1 ${ }^{-/}$tumors in Stat1 ${ }^{+/+}$mice. 
success of the transplantation procedure and to study spontaneous tumorigenesis.

Immunohistochemical staining for STAT1 revealed a successful outgrowth in $36 \%$ of Stat $^{-/}$donor tissue in Stat $^{+/+}$recipient mice upon transplantation, whereas in the Stat $^{+/+}=>$Stat $^{+/+}$setting the take rate reached $90 \%$ (Fig. S1A and S1B). FACS analysis did not show any significant differences in mammary stem cell populations between wild type and Stat $^{-1}$ animals, eliminating the possibility that this cell compartment contributed to the repopulation frequency (Fig. S2A). The analysis of freshly transplanted mammary glands uncovered a dense infiltration with $\mathrm{CD}^{+}$ T-lymphoid cells and NKp46 $6^{+}$NK-cells, irrespective of the genotype (Fig. S2B). Although we used syngenic mice in the experiment - all animals were back-crossed to $\mathrm{BALB} / \mathrm{c}$ - an inflammatory infiltrate is unavoidable due to tissue damage during the transplantation procedure. We

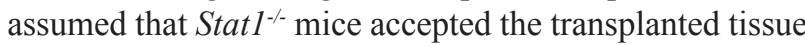
better due to their partially impaired immune system.

Spontaneous tumor development was monitored over a period of 20 months in our cohort of transplanted animals. STAT1 expression was surveyed by immunohistochemical staining and PCR-analysis. Non-transplanted mammary glands where analysed for tumor development and served as an internal control. The observed tumor incidence and latency are summarized in Figs 1E and 1F. Spontaneous tumors developed within 20 months from non-transplanted tissue in 19/35 (54\%) of the Stat $^{-/}$mice and in 4/36 (11\%) of the Stat $^{+/+}$mice. During this time, 3/22 (14\%) of the Stat $^{-1}{ }^{-1}$ animals displayed tumors that originated from the transplanted Stat $1^{+/+}$mammary glands. Even when adjusted for the success rate of transplantation (90\%), the tumor incidence did not exceed $15 \%$ in this group. In the reverse experiment, 3/27 Stat $1^{-/}$tumors evolved in Stat $^{+/+}$mice, translating into a $30 \%$ tumor incidence after adjustment for the significantly lower transplantation success rate.

In summary, our observations enable us to conclude that STAT1 suppresses tumor formation in the mammary epithelial cells themselves. Tumor incidence increases upon transplantation of Stat $^{-/}$mammary glands into Stat $^{+/+}$recipients compared to a Stat $1^{+/+} \Rightarrow$ Stat $^{+/+}$ scenario $(30 \%$ versus $11 \%)$. We also deduce that the STAT1-deficient immune system contributes to and accelerates carcinogenesis. Stat ${ }^{+/+}$as well as Stat1 ${ }^{-1-}$ mammary tumors occurred with an increased incidence in a Stat $1^{-/}$environment compared to in Stat $^{+/+}$surroundings (15\% versus $11 \%$ for Stat $^{+/+}$tumors; $54 \%$ versus 30\% for Stat 1 tumors). Consistently, tumor development occurred with a significantly shorter latency in a Stat $1^{-1-}$ environment, irrespective of whether spontaneous tumor development or carcinomas evolving after transplantation are compared (Fig. 1F).

\section{Loss of STAT1 favors mammary intraepithelial neoplasias (MINs)}

An overview of the histopathological and immunohistochemical features of the tumors that arose in our animal cohort is provided in Table I. Fig. 2A depicts representative panels of the immunohistochemical characterization of two Stat $^{+/+}$and two Stat $1^{-/}$tumors. The tumors were characterized either as neoplasisas in situ (mammary intraepithelial neoplasia, MIN) or as invasive ductal carcinomas. No lobular carcinomas were diagnosed. Grading was performed according to Elston and Ellis [20]. Invasive features were present in $62 \%$ of all Stat $^{-1 /}$ and in $80 \%$ of all Stat $^{+/+}$tumors. We did not detect any genotype-related pattern with regard to grading of tubule formation, nuclear polymorphism or mitotic count. However, we found a high incidence of MIN (8/13) in Stat $1^{-\curvearrowright}$ tumors: 4 of low grade and 4 of high grade (Table I). In contrast, 2/5 Stat $^{+/+}$tumors displayed only lowgrade MINs and no high-grade MIN could be detected. Importantly, the two cases of MIN detected in Stat1 ${ }^{+/+}$ mice had largely lost STAT1 protein expression (Fig. 2B). These observations revealed an increased incidence of MIN upon loss of STAT1.

\section{The STAT1/IRF1 axis is implicated as having an important role in MIN formation, regulating the proliferation of mammary epithelial cells}

Loss of the STAT1 downstream transcription factor IRF1 has been reported in MIN cases of human breast cancer [21]. Moreover, the loss of heterozygosity at the IRF1 gene locus has been found to be a frequent event in human breast cancer [22]. It thus seems possible that STAT1 and IRF1 act in a common axis to suppress MIN and subsequently mammary tumor formation [23, 24]. Initial evidence for this hypothesis was provided by the analysis of STAT1 and IRF1 protein expression in primary mouse mammary tumor tissue: the levels of IRF1 protein correlated with the expression levels of STAT1. Furthermore, IRF1 expression in Stat 1- ${ }^{-1}$ tumors was shown to be low (Fig. 3A). To evaluate whether both Stat $^{1-}$ and $\mathrm{Irfl}^{-/}$mice are predisposed to develop mammary tumors, whole mounts of mammary glands were analyzed. It was noteworthy that already at the virgin state Stat $^{-\leftarrow}$ and Irf ${ }^{-}$ ${ }^{-}$mice displayed an increased amount of ductal structure compared to wild-type controls (Fig. 3B and S3), although MIN could not be detected at that age in these Stat $^{-\digamma}$ and Irf1 ${ }^{-\curvearrowright}$ mice.

To investigate the role of STAT1 and IRF1 in MIN formation, we employed an in vitro three-dimensional culture system, which allowed us to monitor the formation of polarized acini from single mammary epithelial cells [25]. Mammary epithelial cells were prepared from glands of 6- to 8- week-old virgin wild-type, Stat1 ${ }^{-1-}$ 
and $\operatorname{Irf1} 1^{-1}$ mice and analyzed in this $3 \mathrm{D}$ culture system. Acini developed irrespective of the genotype and with no significant difference in total volumes of the spheres (Fig. S4). Remarkably, Stat $1^{-/}$and $\operatorname{Irf1} 1^{-/}$acini displayed a significantly higher cellularity at all time points compared to wild-type controls (Figs 3C, top panel and 3D). Whereas mammospheres of wild-type controls were formed by a single monolayer, immunofluorescent staining showed a less organized cell array and partial epithelial bi-layering in Stat ${ }^{-/}$and $\mathrm{Irfl}^{-/}$spheres (Fig. 3C, bottom panel and Videos S1A, B and C). The increased cellularity could not be attributed to decreased apoptosis as there were no detectable signs of cleaved caspase- 3 activity, even at early time points (day 2) when the lumen of the acini starts to evolve (Fig. S5). This finding is in line with the observations made by Jechlinger [25], who described that lumina form without epithelial cells undergoing apoptosis. The polarity of the Stat $^{-/-}$and $\mathrm{Irfl}^{-/}$acini was unaltered as staining for the basal marker Integrin $\alpha 6$ did not reveal any major changes. Furthermore, we failed to detect any changes in the formation of tight junctions by staining for Zona Occludens-1 (ZO1), a protein that binds directly to occludins and is a bona fide marker for tight junctions (Fig. 3C, bottom panel). However, examining BrdU incorporation revealed a significantly increased proliferation rate in Stat $^{-/-}$and $\operatorname{Irfl}^{-/-}$spheres

A
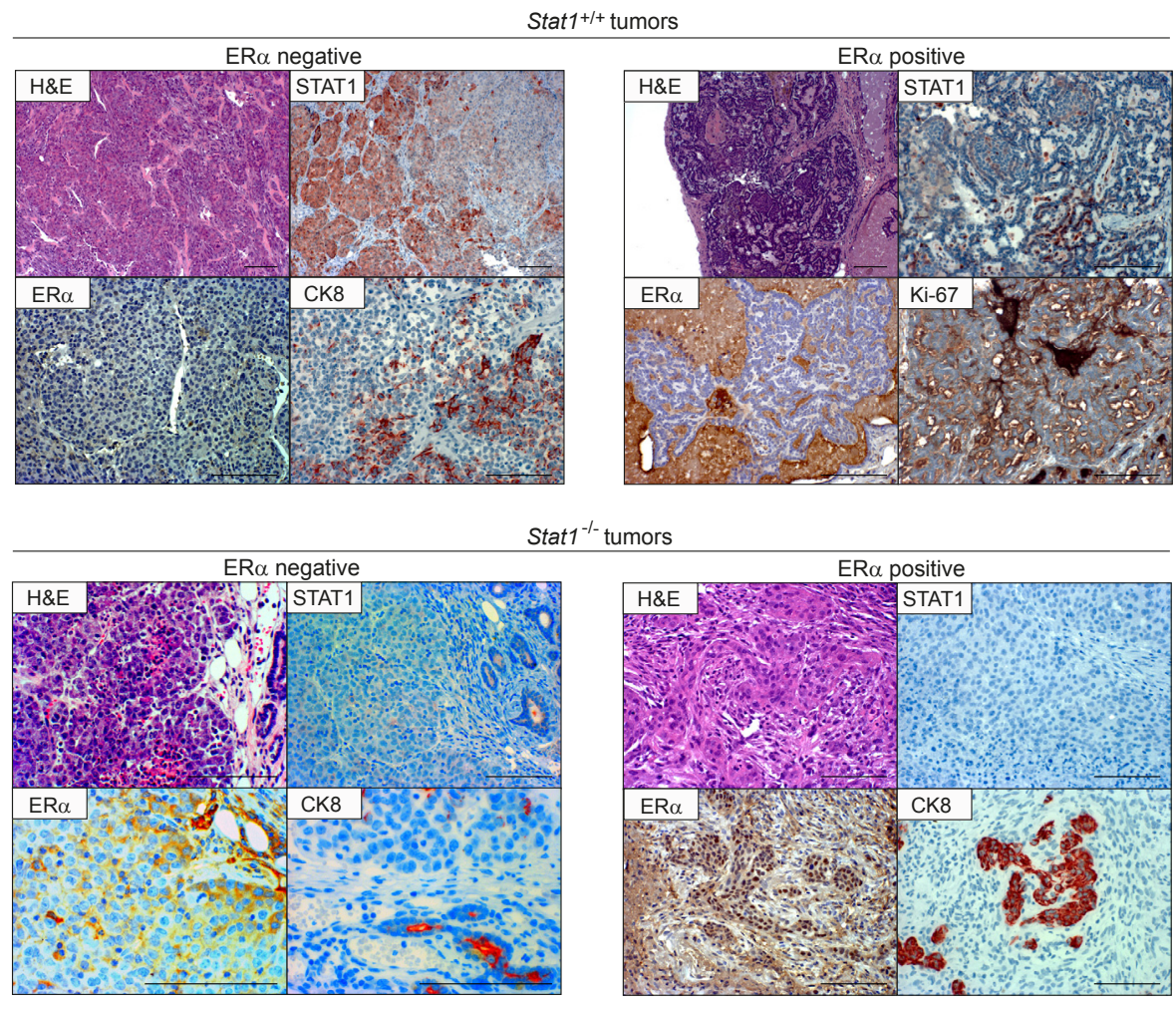

Stat $1^{-1-}$ tumors

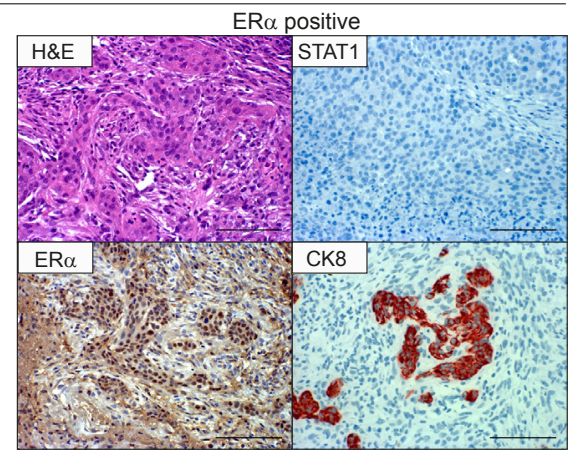

B
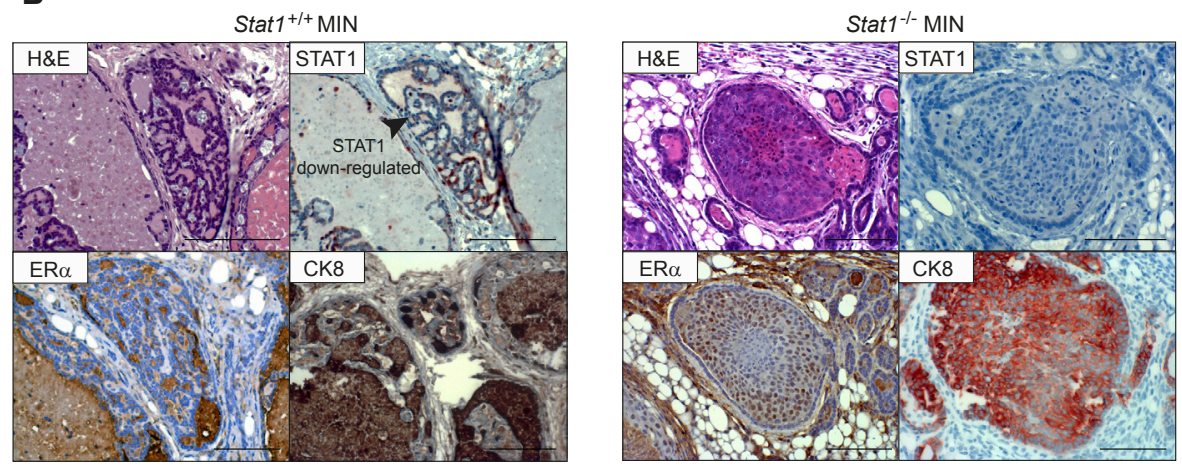

Figure 2: (A) Both, Stat ${ }^{+/+}$and Stat1 $^{-/-}$mammary tumors are heterogenous. Representative pictures of immunohistochemical characterization of two Stat $^{+/+}$(top panel) and two Stat $1^{-/-}$mammary tumors (bottom panel). Scale bars: $100 \mu$ m. (B) Loss of STAT1 correlates with MIN formation. Representative pictures of immunohistochemical characterization of one low-grade Stat $1^{+/+}$(left panel) and one high-grade Stat $^{-/}$MIN (right panel). Stat1 ${ }^{+/+}$MINs display down-regulation of STAT1 protein, as indicated by the arrow. Scale bars: $100 \mu \mathrm{m}$; ER $\alpha$ : estrogen receptor alpha; CK8: cytokeratin 8 . 
A

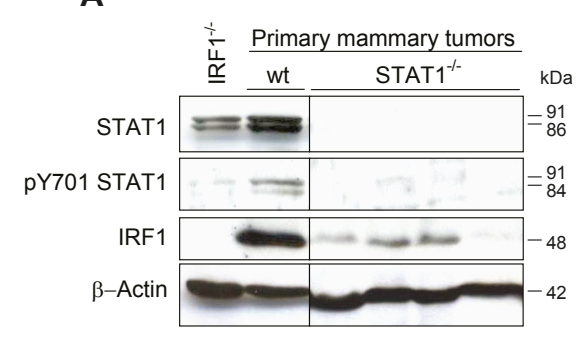

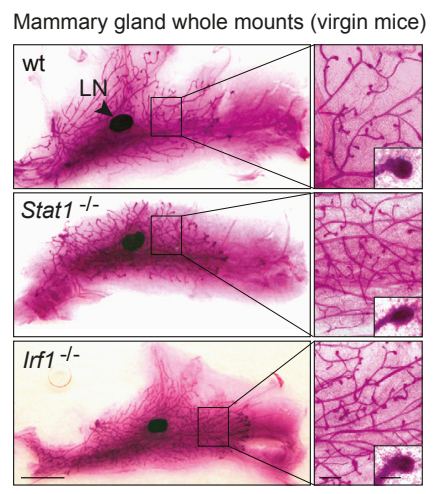

D

C
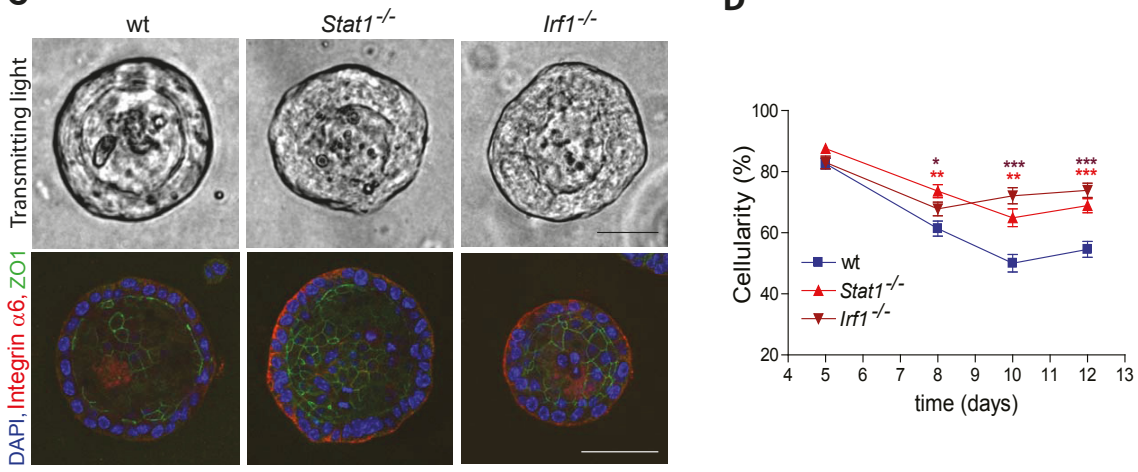

$\mathbf{E}$
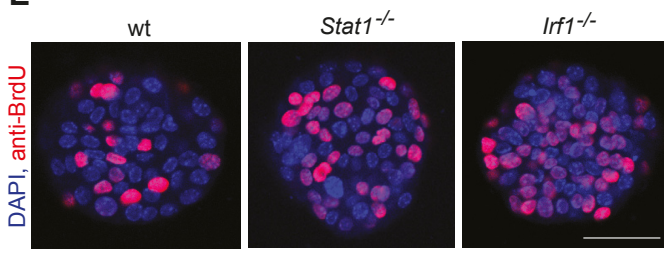

$\mathbf{F}$

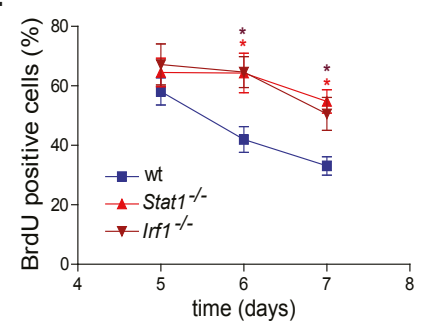

G

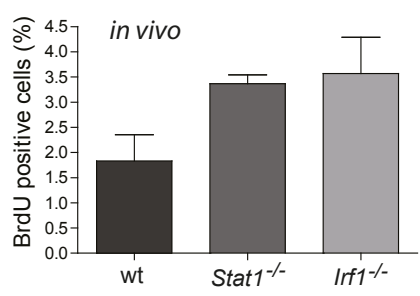

Figure 3: (A, B) Loss of STAT1 might cause mammary tumor formation by down-regulating IRF1. (A) Western blotting of mammary tumor samples. Low expression of IRF1 protein correlates with STAT1 expression. (B) Representative pictures of whole mounts of mammary glands from 50 day-old virgin wild-type (wt), Stat $1^{-/}$and $\operatorname{Irfl}^{-/}$mice. Stat $1^{-/}$and $\operatorname{Irfl}^{-/}$glands show an increased density of ductal structures but no differences in end duct formation. Scale bars: $0.5 \mathrm{~cm}, 500 \mu \mathrm{m}, 100 \mu \mathrm{m}$. LN: lymph node. (C-F) Mammary epithelial cells of Stat $^{-1-}$ and $\mathbf{I r f l}^{-/-}$mice show enhanced proliferation. (C+D) In a 3D culture assay, primary mammary epithelial cells derived from Stat $^{-/}$and $\mathrm{Irfl}^{-/}$mice formed spheres with increased cellularity compared to wild-type controls. (C) Representative brightfield microscopy pictures (top panel) and fluorescence microscopy pictures (bottom panel) from 8 day-old spheres of different genotypes. (Blue) DAPI; (red) Integrin $\alpha 6$; (green) ZO1. Scale bars: $50 \mu \mathrm{m}$. (D) Cellularity of spheres was measured at different time points. For details of calculations see SI Materials and Methods. $n \geq 33$ per genotype. (E+F) To measure proliferation, spheres were exposed to BrdU for 24h, before fixation. (E) Representative fluorescence microscopy pictures of 6 day-old spheres derived from wt, Stat $1^{-/}$and $\operatorname{Irf} 1^{-/-}$epithelial cells. (Blue) DAPI; (red) anti-BrdU. Scale bars: $50 \mu \mathrm{m}$. (F) Percentage of BrdU-positive cells was calculated at different times of growth. $\mathrm{n} \geq 18 ; 1 \mathrm{n}=1$ sphere. (D+F) Statistical analysis: asterisks represent significances to the wild type. No significant differences could be detected between any Stat $1^{-/} / \mathrm{Irfl}^{-/}$pair. Data are representative of three independent experiments. (G) To measure proliferation in vivo, BrdU was injected intraperitoneally into mice. Mammary gland cells were isolated and percentages of BrdU-positive mammary gland cells after 3 days of BrdU administration were measured using the BD FACS-Canto II FACS device with the BD FACS Diva software (Beckton Dickinson). $\mathrm{n}=3$ per genotype. 
compared to wild type controls in vitro (Fig. 3E and 3F). This was confirmed by analysis of mammary epithelial proliferation in vivo. For this purpose, BrdU was injected intraperitoneally into mice for a period of 3 days. Isolated mammary glands were digested and cells in singlecell suspensions were stained with anti-BrdU antibody. Quantification of BrdU-positive cells by flow cytometry confirmed the enhanced proliferation of Stat $1^{-/}$and $\mathrm{Irfl}^{-/-}$ mammary epithelial cells (Fig. 3G).

\section{Mammary tumor formation is under the control of cytotoxic T-lymphocytes (CTLs)}

Our transplantation studies revealed that the absence of STAT1 from the immune system significantly enhances tumor incidence and shortens the latency of mammary tumor formation. STAT1 is believed to have an essential role in CTL- and NK-cell cytotoxicity; both lymphoid lineages are important mediators of tumor surveillance. Immunohistochemical staining for CD3 and NKp46 verified a dense infiltration of all mammary tumors with cytotoxic T-cells, whereas in all tumors NK-cells were rare and not consistently detectable (Fig. 4A). To clarify the contribution of NK and/or cytotoxic $\mathrm{T}$ cells to tumor surveillance, we generated mammary tumor cell lines. Four cell lines (\#1, \#2: Stat1 ${ }^{-/}$; \#3, \#4: Stat1 ${ }^{+/+}$) were established during the course of our study, all of which displayed an epithelial-like phenotype (Fig. 4B). Of note, cell lines lacking STAT1 had a proliferative advantage in vitro and in vivo over Stat $^{+/+}$lines (Fig. 4C and S6A-C). $5 \times 10^{5}$ cells were orthotopically injected into the mammary

Table I: Histopathological and immunohistochemical analysis and classification of mammary tumors. Histological sections of all available mammary tumors were analyzed and mouse mammary tumors characterized according to a standard nomenclature used to classify human breast carcinomas. Invasive carcinomas were graded according to Elson and Ellis [20]. 1: well-differentiated breast cells, cells generally appear normal and do not grow rapidly, cancer arranged in small tubules; 2: moderately-differentiated breast cells, have characteristics between Grade 1 and Grade 3 tumors; 3: poorly differentiated breast cells, cells do not appear normal and tend to grow and spread more aggressively. Tubule formation ( $\%$ of carcinoma composed of tubular structures) - 1: $>75 \% ; 2: 10-75 \% ; 3:$ less than $10 \%$. Nuclear pleomorphism - 1: small, uniform cells; 2: moderate increase in size and variation; 3: marked variation. Mitosis count - 1: up to 7; 2: 8 to 14; 3: 15 or more. Mammary intraepithelial neoplasia (MIN) - -: not detected; low grade; moderate grade; high grade. Estrogen receptor alpha $(\mathrm{ER} \alpha)$ - Reiner score [36], i.e. 0-2: negative; 3: low positive; 4-5: moderate positive; 6-7: strong positive. Human epidermal growth factor receptor 2 (HER2) - 0-1: negative; 2: low positive; 3: strong positive.

\begin{tabular}{|c|c|c|c|c|c|c|c|c|c|c|}
\hline \multirow[b]{2}{*}{ Number } & \multirow{2}{*}{$\begin{array}{l}\text { Genotype } \\
\text { Tumor }\end{array}$} & \multirow{2}{*}{$\begin{array}{l}\text { Genotype } \\
\text { Mouse }\end{array}$} & \multirow[b]{2}{*}{ Histological Classification } & \multicolumn{4}{|c|}{ Invasiveness } & \multirow[b]{2}{*}{ MIN } & \multirow[b]{2}{*}{ ERa } & \multirow[b]{2}{*}{ HER2 } \\
\hline & & & & $\begin{array}{l}\text { Tubule } \\
\text { formation }\end{array}$ & $\begin{array}{c}\text { Nuclear } \\
\text { pleomorphism }\end{array}$ & $\begin{array}{l}\text { Mitosis } \\
\text { count }\end{array}$ & Grading & & & \\
\hline 1 & Stat $1 \%$ & Stat $1 \%$ & ductal/with medullary features & 3 & 3 & 3 & 3 & - & 0 & 0 \\
\hline 2 & Stat $1^{-/}$ & Stat $1^{-1 /}$ & ductal & 2 & 2 & 1 & 1 & - & 0 & 0 \\
\hline 3 & Stat $1^{-}$ & Stat $1^{-1}$ & ductal & 3 & 3 & 3 & 3 & - & 0 & 0 \\
\hline 4 & Stat $1^{-/}$ & Stat $1^{1 /}$ & ductal & 2 & 3 & 3 & 3 & high grade & $4(60 \% / 2)$ & 1 \\
\hline 5 & Stat $1 \%$ & Stat $1 \%$ & ductal & 3 & 3 & 3 & 3 & high grade & $3(30 \% / 1)$ & 0 \\
\hline 6 & Stat $1^{-}$ & Stat $1 \%$ & $\begin{array}{c}\text { ductal/with metaplastic } \\
\text { f eatures }\end{array}$ & 3 & 3 & 2 & 3 & high grade & $3(20 \% / 1)$ & 0 \\
\hline 7 & Stat $1^{-/}$ & Stat $1^{-/}$ & intraductal with microinvasion & & & & & low grade & 0 & $1 \sim 2$ \\
\hline 8 & Stat $1^{\%}$ & Stat $1^{-/}$ & intraductal-papillary & & & & & low grade & $3(10 \% / 1)$ & 0 \\
\hline 9 & Stat $1^{-1}$ & Stat $1^{-1}$ & intraductal with microinvasion & & & & & low grade & $3(50 \% / 1)$ & 0 \\
\hline 10 & Stat ${ }^{\%}$ & Stat $1^{-1}$ & Intraductal-papillary & & & & & low grade & 0 & 0 \\
\hline 11 & Stat $1^{-/}$ & Stat $1^{+/+}$ & ductal & 2 & 2 & 1 & 1 & - & 0 & 1 \\
\hline 12 & Stat $1^{-}$ & Stat $1^{+/+}$ & ductal & 3 & 3 & 2 & 3 & - & $4(60 \% / 2)$ & 0 \\
\hline 13 & Stat $1^{-/}$ & Stat $1^{+/+}$ & intraductal & & & & & high grade & $4(60 \% / 2)$ & 1 \\
\hline 14 & Stat $1^{+/+}$ & Stat $1^{-/}$ & $\begin{array}{c}\text { ductal/with metaplastic } \\
\text { f eatures }\end{array}$ & 3 & 3 & 2 & 3 & - & $0(<10 \%)$ & 0 \\
\hline 15 & Stat $1^{+/+}$ & Stat $1^{+/+}$ & ductal & 1 & 1 & 1 & 1 & - & $5(50 \% / 3)$ & 2 \\
\hline 16 & Stat $1^{+/+}$ & Stat $1^{+/+}$ & ductal & 2 & 2 & 3 & 2 & - & 0 & 1 \\
\hline 17 & Stat $1^{+/+}$ & Stat $1^{+/+}$ & ductal & 1 & 1 & 1 & 1 & low grade & $0(<10 \%)$ & 0 \\
\hline 18 & Stat $1^{+/+}$ & Stat $1^{+/+}$ & intraductal with microinvasion & & & & & low grade & 0 & n.a. \\
\hline
\end{tabular}


glands of wild type, Stat1 $1^{-/}$, Rag2 $2^{-/-}$or Stat $1^{-/}$Rag2 $^{-/-}$mice. As Rag2 ${ }^{--}$animals lack T cells and rely on NK cells for tumor surveillance, this experiment allowed us to determine the individual contribution of $\mathrm{T}$ and $\mathrm{NK}$ cells to tumor surveillance. The experiment was terminated and tumor weights determined when the first tumors reached approximately $1 \mathrm{~cm}$ in diameter. The Stat $^{1 /-}$ cell line \#2 failed to induce tumors in wild-type mice and tumor formation was restricted to immuno-compromised animals. This observation is consistent with previous findings that tumors evolving in immunodeficient hosts (such as Stat1 ${ }^{-/}$) are more immunogenic and not immunoedited and may be rejected in immuno-competent surroundings [26].

Fig. 4D summarizes the data from orthotopic tumor injections. We found a consistently and significantly enhanced tumor growth in Stat $^{-/-}$mice compared to wild-type controls, irrespective of the cell line injected. Compared to $\mathrm{Rag}^{-/}$animals, tumor growth in $\mathrm{Stat1}^{-/}$ or Stat $^{-/}$Rag2 $2^{-/}$hosts was not significantly accelerated, indicating that the NK-cell compartment played at most a minor part in restricting proliferation of the transplanted tumor cells. Support for this conclusion came from a comparison of wild-type recipients with $\operatorname{Rag}^{-/}$mice. Although $\mathrm{Rag}^{-/}$mice contain functionally competent NK-cells, tumor growth in these mice was significantly increased, indicating that the NK cell compartment was not capable of significantly limiting tumor expansion. In summary, the data indicate a dominant role for cytotoxic $\mathrm{T}$ cells in tumor surveillance in spontaneously occurring, parity-induced mammary tumors.
A

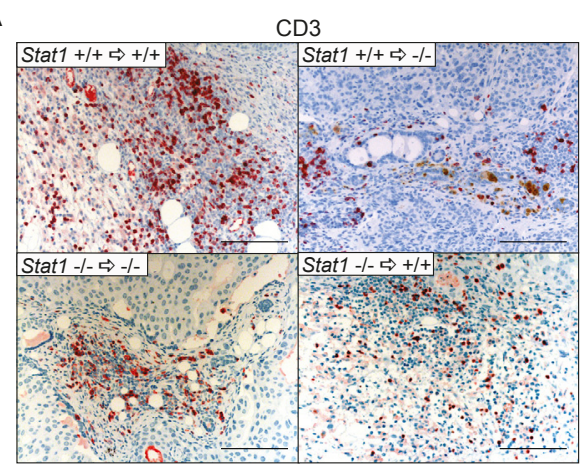

B Mammary tumor cell lines:

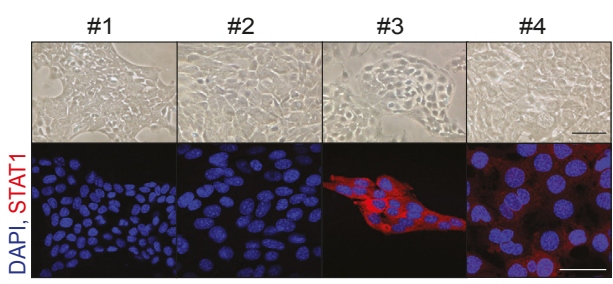

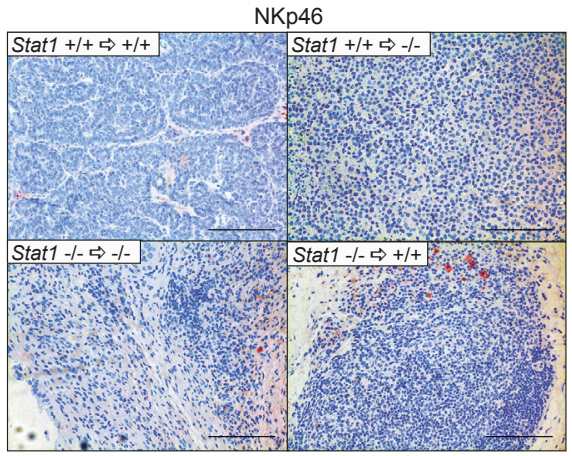

C Growth curve:

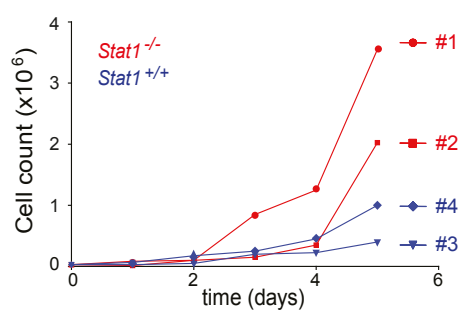

Orthotopic injection:
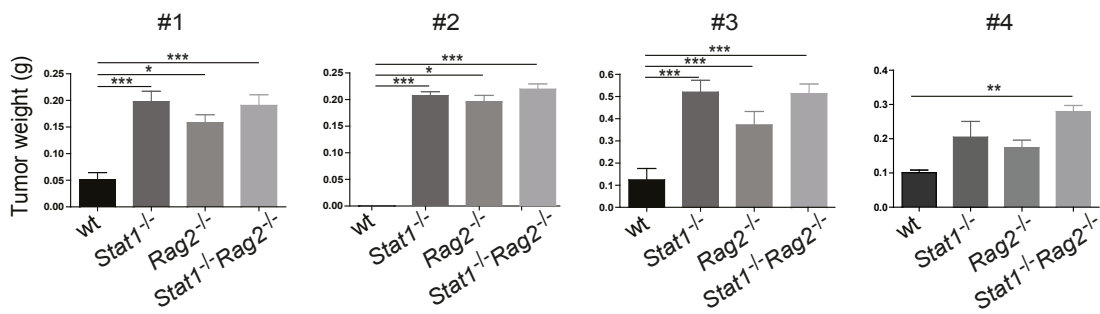

Figure 4: Mammary tumor growth is mainly controlled by cytotoxic T-cells and only to a minor extent by NK-cells. (A) Histological sections of spontaneously occurring mammary tumors of the transplant experiment were stained with CD3 and NKp46 antibodies. Tumors are listed in four groups: Stat $1^{+/+}$tumors in Stat ${ }^{+/+}$mice, Stat $1^{+/+}$tumors in Stat $1^{-/-}$mice, Stat $1^{-/-}$tumors in Stat1 $1^{-/-}$mice and Stat $1^{-/-}$tumors in Stat ${ }^{+/+}$mice. Scale bars: $100 \mu \mathrm{m}$. (B) Bright-field (top panel) and fluorescence (bottom panel) microscopy pictures of tumor cell lines derived from two Stat $1^{-/}(\# 1, \# 2)$ and two Stat $^{+/+}$(\#3, \#4) spontaneous mammary tumors. (Blue) DAPI; (red) STAT1. Scale bars: $50 \mu \mathrm{m}$. (C) Growth curve of mammary tumor cell lines. (D) Mammary tumor cell lines were orthotopically injected into wt, Stat $^{-/}$, Rag $2^{-/}$and Stat 1 ${ }^{-/}$Rag $^{2-/}$ animals. Tumor weights of different groups were compared. $\mathrm{n} \geq 5$ per genotype. All data are representative of two independent experiments. 


\section{DISCUSSION}

We show that the transcription factor STAT1 has a tumor-suppressing function against the formation of parity-induced, spontaneous mammary tumors. We show that STAT1 deficiency significantly increases tumor incidence in BALB/c mice as well as decreasing disease latency. STAT1 acts in a dual manner. It sustains proper CTL activity and thus ensures tumor surveillance, while also exerting growth inhibitory and tumor suppressing effects via its downstream regulator IRF1.

Our findings are in line with recent reports on the role of STAT1 in the context of ERBB2/neu/HER2 induced mammary cancer development $[9,10]$. Using different mouse models, both groups concluded that STAT1 suppresses ERBB2/neu/HER2 tumor formation. In our cohort of mice we characterized tumors that evolved spontaneously in the absence of a driving oncogene. The fact that only $2 / 13$ of the mammary cancers expressed ERBB2/neu/HER2 indicates that STAT1's tumor-suppressing effect is not limited to ERBB2/neu/ HER2-induced tumorigenesis. The effect of STAT1 loss is also not restricted to tumors that display a particular pattern of expression of the estrogen receptor (ER): 7/13 of the spontaneously evolving Stat $1^{-/}$mammary cancers were $\mathrm{ER}^{+}$, while the remaining cases did not express detectable levels of the ER protein. These findings show conclusively that STAT1 is a global tumor suppressor that acts independently of a distinct oncogenic driver.

The importance of STAT1 as a tumor suppressor was underlined by the finding that all Stat ${ }^{+/+}$mammary cancers had partially lost or down-regulated STAT1 protein expression. Similar observations were reported in human patients, where low levels of STAT1 activation have been linked to a poor prognosis [5, 27]. The selection pressure to down-regulate or lose STAT1 in tumor cells may have more than one cause as the loss of STAT1 has several important consequences: the tumor cells lose responsiveness towards interferon-mediated growth inhibition (interferons are important players in tumor surveillance). Moreover, STAT1 is the key regulator of MHC class I expression. By losing STAT1 the cells may down-regulate MHCI and thus escape CTL-mediated tumor surveillance $[28,29]$. As NK cells play a negligible part in tumor surveillance, MHCI loss represents a clearcut advantage. CTLs dominated the immune infiltrate in the tumor sections and their importance was further verified in transplant studies. In the absence of the adaptive immune system, tumor development occurred rapidly and was significantly accelerated, whereas the presence of NK cells did not interfere with tumor onset. Hence, mammary tumors are partially able to escape immune control by down-regulating STAT1. In Stat1 ${ }^{-1}$ animals, the selective pressure to escape immune control is less important as the mice have several defects that limit their capability to exert effective tumor surveillance. As a consequence, mammary cancer formation is increased in a Stat $1^{-/}$micro-environment, irrespective of whether the epithelial cells themselves express STAT1. The finding underlines the role of the immune system in spontaneously evolving mammary cancer. Although a pro-inflammatory environment and T-cell infiltration may exert a tumor promoting effect, our orthotopic injection experiments unequivocally show that there is a tumor suppressing function that opposes the tumor promoting effect of the immune infiltrate.

Loss or down-regulation of STAT1 confers an additional advantage, i.e. accelerated cell proliferation. Under normal conditions, STAT1 puts the brakes on cell proliferation in mammary epithelial cells, presumably via the transcription factor IRF1. Two lines of evidence support this assumption. First, whole mounts of Stat $1^{-/}$as well as of $\mathrm{Irfl}^{-/}$mammary glands display increased amounts of ductal structures compared to wild type controls, even in the virgin state. Secondly, BrdU incorporation studies in vitro and in vivo confirm an increased DNA synthesis and thus enhanced growth in mammary epithelial cells of both Stat ${ }^{-\leftarrow}$ and $\operatorname{Irf} 1^{-\leftarrow}$ mice.

As a consequence of enhanced cell proliferation, MIN formation was frequently found in Stat $1^{-1}$ mice. This finding was also reflected in 3D-culture experiments, in which the cell composition of Stat $1^{-/}$and $\operatorname{Irfl}^{-/}$acini was less organized. The original report describing the 3D system used the two oncogenes MYC and Kras ${ }^{G 12 D}$ to characterize the occurrence of highly proliferative depolarized spheres resembling MIN. As expected, the alterations observed in Stat $^{-\%}$ - and $\mathrm{Irfl}^{-\%}$-derived spheres are less pronounced than those occurring in the presence of MYC and $\mathrm{Kras}^{G 12 D}$. In the absence of STAT1 and IRF1, polarity of the spheres is preserved and a lumen albeit smaller - is maintained in most cases. Therefore, additional alterations are required to allow mammary tumor formation. These additional alterations are most probably triggered by hormonal stimulation and changes occurring during lactation in the breast tissue, as we failed to detect spontaneous tumorigenesis in nulliparous mice. Our conclusion that STAT1/IRF1 act in a linear axis to block growth is in perfect accordance with reports by others that attribute a negative regulatory role of IRF1 on cell growth: enforced expression of IRF1 in mammalian cell lines slows or even halts proliferation [30-32].

Cancer formation is a multi-level process during which a cell successively acquires several genetic or epigenetic alterations that ultimately cooperate to allow the development of a malignant tumor. One of the alterations required to initiate the process is loss of growth control. Our findings support a model in which STAT1 represents a critical safeguard that preserves growth control in mammary epithelial cells. The absence of STAT1 facilitates cell proliferation and therefore MIN 
formation, which represents the first step on the road from normal breast tissue towards invasive breast cancer [33].

The exact characterization of the early alterations has a significant potential for use in preventative therapy of invasive breast cancer and might lead to the development of novel immune-modulatory strategies to combat the disease. The critical effect of STAT1 is not restricted to any distinct tumor type but is of global relevance.

\section{MATERIAL AND METHODS}

Mice

All animals were maintained in spf quality at the University of Veterinary Medicine, Vienna. C.Cg-Stat $1^{\mathrm{tm} 1}$ (Stat1 $1^{-/}$) [16], C.Cg-Irf1 ${ }^{\mathrm{tm} 1 \mathrm{Mak}}$ (Irf1 ${ }^{-/}$) [34] and C.129S6Rag2 ${ }^{\text {tmlFwa }}\left(\operatorname{Rag}^{-/}\right)$[35] mice have been described previously. C.Cg-Stat1 ${ }^{\mathrm{tm} 1}-\mathrm{Rag} 2^{\mathrm{tm} 1 \mathrm{Fwa}}\left(\right.$ Stat $^{-/}$Rag2 $\left.^{-/-}\right)$mice were crossed at the University of Veterinary Medicine, Vienna. Animal experiments were discussed and approved by the institutional ethics committee and undertaken in conformance with Austrian laws.

\section{Orthotopic injection of mammary tumor cell lines}

Mammary tumor cell lines \#1-\#4 were derived from spontaneous mammary tumors. For orthotopic injection of mammary tumor cell lines, mice were anaesthetized and depilated on the belly. $5 \times 10^{5}$ cells were injected via the nipple into the fat pad of the $4^{\text {th }}$ and $5^{\text {th }}$ mammary gland of wild type, Stat $1^{-/}$, Rag2 $2^{-/}$and Stat $1^{-/}$Rag $^{-/-}$mice. The mice were sacrificed when the tumors reached one centimeter in diameter.

\section{Histology and immunohistochemistry}

The following antibodies were used for immunostaining in accordance with to the manufacturers' protocol: STAT1 (Santa Cruz, \#sc-592), CD3 (Neomarkers, RM9107), NKp46 (BioLegend, \#137601), ER $\alpha$ (Santa Cruz, \#sc-542), CK8 (Developmental Studies Hybridoma Bank, TROMA-I) and Ki67 (Novocastra, NCL-Ki67). Nuclear counterstaining was performed with hematoxylin. Pictures were taken on a Zeiss AxioImager Z1 microscope system with a CCD camera using the software PixelNK Capture 3.0.

\section{Immunofluorescence}

For immunofluorescent staining, cells or 3D cultures were incubated with primary antibodies against STAT1 (Santa Cruz, \#sc-592), integrin a6 (Millipore, \#MA1378) or ZO1 (Zymed Laboratories, \#33-9100), followed by incubation with Alexa546-conjugated or Alexa488conjugated goat antibodies against rat or mouse IgG (Molecular Probes, \#A11081 and \#A11001). Incubation times were prolonged for $3 \mathrm{D}$ cultures to ensure complete staining. Cells were counterstained with DAPI and imaged using a confocal laser-scanning microscope (Carl Zeiss LSM 700, Occulare 10x, 40x Oil) using the software ZEN 2009 LE.

\section{Statistics}

All statistical analysis was performed using GraphPad Prism 4. Differences were assessed for statistical significance by One-way ANOVA using the Tukey's Multiple Comparison Test. For Figure 1B only, the unpaired t-test was used. Error bars represent mean \pm SD. $P$ values are considered as follows: $<0.05: * ;<0.01$ : $* * ;<0.001$ : ***.

\section{ACKNOWLEDGEMENTS}

We thank M. Gröger for help and support with confocal microscopy and S. Fajmann and M. Schlederer for technical assistance with histo-pathology. We are indebted to S. Gratzl and the team of the Institute of Animal Breeding and Genetics and Biomodels Austria (BIAT) for taking excellent care of the mice. We are grateful to $\mathrm{R}$. Moriggl, A. Jamieson, B. Strobl and all members of the SFB 28 for helpful discussions.

This work was supported by the Austrian Science Fund (FWF) by a grant to VS, TD and MM (SFB F28) and to DS (19534), the Austrian Federal Ministry of Science and Research by grant BM_WFa GZ200.112/1-VI/1/2004, GZ BMWF-200.191/1-II/1a/2008 to $\mathrm{MM}$ and the BM WFa program GEN-AU II and III project Austromouse to MM. K.-U. Wagner is supported by a Public Health Service Grant (CA117930) from the National Cancer Institute. HD is supported by the Herzfelder Family Foundation and Life Science Krems.

\section{REFERENCES}

1. Yu H, Pardoll D, Jove R. STATs in cancer inflammation and immunity: a leading role for STAT3. Nature reviews. 2009; 9:798-809.

2. Catlett-Falcone R, Dalton WS, Jove R. STAT proteins as novel targets for cancer therapy. Signal transducer an activator of transcription. Current opinion in oncology. 1999; 11:490-496.

3. Watson CJ, Neoh K. The Stat family of transcription factors have diverse roles in mammary gland development. Semin Cell Dev Biol. 2008; 19:401-406.

4. Philp JA, Burdon TG, Watson CJ. Differential activation of STATs 3 and 5 during mammary gland development. 
FEBS Lett. 1996; 396:77-80.

5. Widschwendter A, Tonko-Geymayer S, Welte T, Daxenbichler G, Marth C, Doppler W. Prognostic significance of signal transducer and activator of transcription 1 activation in breast cancer. Clin Cancer Res. 2002; 8:3065-3074.

6. Yau C, Esserman L, Moore DH, Waldman F, Sninsky J, Benz CC. A multigene predictor of metastatic outcome in early stage hormone receptor-negative and triple-negative breast cancer. Breast Cancer Res. 2010; 12:R85.

7. Thomas M, Finnegan CE, Rogers KM, Purcell JW, Trimble A, Johnston PG, Boland MP. STAT1: a modulator of chemotherapy-induced apoptosis. Cancer Res. 2004; 64:8357-8364.

8. Yang PY, Chen MF, Kao YH, Hu DN, Chang FR, Wu YC. Norcantharidin induces apoptosis of breast cancer cells: Involvement of activities of mitogen activated protein kinases and signal transducers and activators of transcription. Toxicol In Vitro. 2011.

9. Klover PJ, Muller WJ, Robinson GW, Pfeiffer RM, Yamaji D, Hennighausen L. Loss of STAT1 from mouse mammary epithelium results in an increased neu-induced tumor burden. Neoplasia. 2010; 12:899-905.

10. Raven JF, Williams V, Wang S, Tremblay ML, Muller WJ, Durbin JE, Koromilas AE. Stat1 is a suppressor of ErbB2/ Neu-mediated cellular transformation and mouse mammary gland tumor formation. Cell Cycle. 2011; 10:794-804

11. Koch JG, Gu X, Han Y, El-Naggar AK, Olson MV, Medina D, Jerry DJ, Blackburn AC, Peltz G, Amos CI, Lozano G. Mammary tumor modifiers in BALB/cJ mice heterozygous for p53. Mamm Genome. 2007; 18:300-309.

12. Townsend PA, Scarabelli TM, Davidson SM, Knight RA, Latchman DS, Stephanou A. STAT-1 interacts with p53 to enhance DNA damage-induced apoptosis. J Biol Chem. 2004; 279:5811-5820.

13. Lee CK, Smith E, Gimeno R, Gertner R, Levy DE. STAT1 affects lymphocyte survival and proliferation partially independent of its role downstream of IFN-gamma. J Immunol. 2000; 164:1286-1292.

14. Stephanou A, Scarabelli TM, Brar BK, Nakanishi Y, Matsumura M, Knight RA, Latchman DS. Induction of apoptosis and Fas receptor/Fas ligand expression by ischemia/reperfusion in cardiac myocytes requires serine 727 of the STAT-1 transcription factor but not tyrosine 701 . J Biol Chem. 2001; 276:28340-28347.

15. Kim HS, Lee MS. STAT1 as a key modulator of cell death. Cellular signalling. 2007; 19:454-465.

16. Durbin JE, Hackenmiller R, Simon MC, Levy DE. Targeted disruption of the mouse Stat1 gene results in compromised innate immunity to viral disease. Cell. 1996; 84:443-450.

17. Meraz MA, White JM, Sheehan KC, Bach EA, Rodig SJ, Dighe AS, Kaplan DH, Riley JK, Greenlund AC, Campbell D, Carver-Moore K, DuBois RN, Clark R, Aguet M, Schreiber RD. Targeted disruption of the Stat1 gene in mice reveals unexpected physiologic specificity in the JAKSTAT signaling pathway. Cell. 1996; 84:431-442.

18. Pilz A, Kratky W, Stockinger S, Simma O, Kalinke U, Lingnau K, von Gabain A, Stoiber D, Sexl V, Kolbe T, Rulicke T, Muller M, Decker T. Dendritic cells require STAT-1 phosphorylated at its transactivating domain for the induction of peptide-specific CTL. J Immunol. 2009; 183:2286-2293.

19. Lee CK, Rao DT, Gertner R, Gimeno R, Frey AB, Levy DE. Distinct requirements for IFNs and STAT1 in NK cell function. J Immunol. 2000; 165:3571-3577.

20. Elston CW, Ellis IO. Pathological prognostic factors in breast cancer. I. The value of histological grade in breast cancer: experience from a large study with long-term follow-up. Histopathology. 1991; 19:403-410.

21. Doherty GM, Boucher L, Sorenson K, Lowney J. Interferon regulatory factor expression in human breast cancer. Ann Surg. 2001; 233:623-629.

22. Cavalli LR, Riggins RB, Wang A, Clarke R, Haddad BR. Frequent loss of heterozygosity at the interferon regulatory factor-1 gene locus in breast cancer. Breast Cancer Res Treat. 2009; 121:227-231.

23. Kim PK, Armstrong M, Liu Y, Yan P, Bucher B, Zuckerbraun BS, Gambotto A, Billiar TR, Yim JH. IRF1 expression induces apoptosis and inhibits tumor growth in mouse mammary cancer cells in vitro and in vivo. Oncogene. 2004; 23:1125-1135.

24. Bouker KB, Skaar TC, Riggins RB, Harburger DS, Fernandez DR, Zwart A, Wang A, Clarke R. Interferon regulatory factor-1 (IRF-1) exhibits tumor suppressor activities in breast cancer associated with caspase activation and induction of apoptosis. Carcinogenesis. 2005; 26:15271535 .

25. Jechlinger M, Podsypanina K, Varmus H. Regulation of transgenes in three-dimensional cultures of primary mouse mammary cells demonstrates oncogene dependence and identifies cells that survive deinduction. Genes Dev. 2009; 23:1677-1688.

26. Shankaran V, Ikeda H, Bruce AT, White JM, Swanson PE, Old LJ, Schreiber RD. IFNgamma and lymphocytes prevent primary tumour development and shape tumour immunogenicity. Nature. 2001; 410:1107-1111.

27. Charpin C, Secq V, Giusiano S, Carpentier S, Andrac L, Lavaut MN, Allasia C, Bonnier P, Garcia S. A signature predictive of disease outcome in breast carcinomas, identified by quantitative immunocytochemical assays. International journal of cancer. 2009; 124:2124-2134.

28. Ward PL, Schreiber H. MHC class I restricted T cells and immune surveillance against transplanted ultraviolet lightinduced tumors. Seminars in cancer biology. 1991; 2:321328.

29. Philipps C, Stauss HJ, Wortzel RD, Schreiber H. A novel MHC class I molecule as a tumour-specific antigen. Correlation between the antibody-defined and the CTL- 
defined target structure. Journal of immunogenetics. 1986; 13:93-99.

30. Kirchhoff S, Schaper F, Hauser H. Interferon regulatory factor 1 (IRF-1) mediates cell growth inhibition by transactivation of downstream target genes. Nucleic Acids Res. 1993; 21:2881-2889.

31. Romeo G, Fiorucci G, Chiantore MV, Percario ZA, Vannucchi S, Affabris E. IRF-1 as a negative regulator of cell proliferation. J Interferon Cytokine Res. 2002; 22:3947.

32. Taniguchi T, Harada H, Lamphier M. Regulation of the interferon system and cell growth by the IRF transcription factors. Journal of cancer research and clinical oncology. 1995; 121:516-520.

33. Virnig BA, Tuttle TM, Shamliyan T, Kane RL. Ductal carcinoma in situ of the breast: a systematic review of incidence, treatment, and outcomes. J Natl Cancer Inst. 2010; 102:170-178.

34. Matsuyama T, Kimura T, Kitagawa M, Pfeffer K, Kawakami T, Watanabe N, Kundig TM, Amakawa R, Kishihara K, Wakeham A, et al. Targeted disruption of IRF-1 or IRF-2 results in abnormal type I IFN gene induction and aberrant lymphocyte development. Cell. 1993; 75:83-97.

35. Shinkai Y, Rathbun G, Lam KP, Oltz EM, Stewart V, Mendelsohn M, Charron J, Datta M, Young F, Stall AM, et al. RAG-2-deficient mice lack mature lymphocytes owing to inability to initiate V(D)J rearrangement. Cell. 1992; 68:855-867.

36. Regitnig P, Reiner A, Dinges HP, Hofler G, Muller-Holzner E, Lax SF, Obrist P, Rudas M, Quehenberger F. Quality assurance for detection of estrogen and progesterone receptors by immunohistochemistry in Austrian pathology laboratories. Virchows Arch. 2002; 441:328-334. 